\title{
A Feedback Paradigm for Latent Fingerprint Matching
}

\author{
Eryun Liu, Sunpreet S. Arora, Kai Cao and Anil K. Jain \\ Department of Computer Science and Engineering \\ Michigan State University \\ East Lansing, MI, U.S.A. \\ \{liueryun, arorasun, kaicao, jain\}@cse.msu.edu
}

\begin{abstract}
Latent fingerprints are of critical value in forensic science because they serve as an important source of evidence in a court of law. Automatic matching of latent fingerprints to rolled/plain (exemplar) fingerprints with high accuracy is quite vital for such applications. However, due to poor latent image quality in general, latent fingerprint matching accuracy is far from satisfactory. In this research, we propose a novel latent matching paradigm which takes feedback from an exemplar print during matching to refine the features extracted from the latent. The refined latent features are then used to update the baseline match scores and resort the candidate list retrieved from the database. Experimental results show that the feedback based matching mechanism improves the rank-1 identification accuracy of the baseline latent matcher by about $8 \%$ and $3 \%$ for NIST SD27 and WVU latent databases, respectively. The proposed feedback paradigm can be wrapped around any latent matcher to improve its performance.
\end{abstract}

\section{Introduction}

Ever since the uniqueness and individuality of the ridge valley fingerprint patterns was first claimed by Sir Galton in 1892 [11], fingerprint has been one of the most extensively used biometric modalities for identifying individuals. While fingerprint recognition has found applications in both civilian as well as law enforcement scenarios, it is the law enforcement agencies that have successfully used it for identifying suspects for almost 100 years [5]. There are typically three different kinds of fingerprints encountered in such applications: (a) Rolled fingerprints which contain almost all of the ridge details and are acquired by rolling a finger from nail-to-nail, (b) Plain/Slap fingerprints which are obtained by pressing the finger on a flat surface, and (c) Latent fingerprints which are impressions left on the surface of objects touched or handled by fingers. Latent fingerprints, in particular are of critical value in forensic applica-

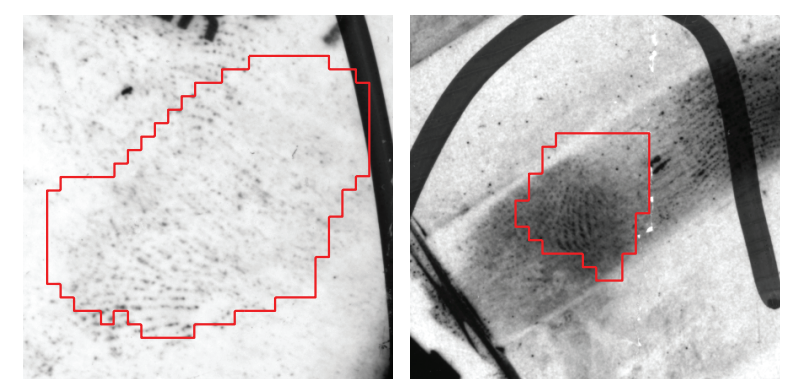

Figure 1. Sample images from NIST SD27 database to elucidate some of the challenges in latent fingerprint matching: (a) poor ridge clarity in the latent and (b) insufficient amount of usable ridge valley patterns. The red curves indicate the manually marked foreground area in the image.

tions because they are usually encountered at crime scenes and serve as crucial evidence in a court of law.

Rolled and plain impressions (exemplars ${ }^{1}$ ), acquired under supervision, are mostly good quality fingerprints with sufficient amount of ridge patterns and clear ridge structures, and typically contain only minor background noise. Latent fingerprints, on the other hand, are partial impressions of the finger either captured photographically or lifted from objects using complex chemical or dusting procedures [15] [5]. Usually, latents contain only a small amount of usable ridge structures and exhibit poor quality in terms of ridge clarity due to the presence of background noise (Figure 1). They may also have large non-linear distortions due to variations in finger pressure when the object is being touched and the print is left on its surface.

The results of Fingerprint Vendor Technology Evaluation (FpVTE) [23] conducted by NIST showed that the best performing commercial matcher achieved rank-1 identification rate of more than $99.4 \%$ on a database of 10,000 plain fingerprint images. On the other hand, in another multiphase project on Evaluation of Latent Fingerprint Technolo-

\footnotetext{
${ }^{1}$ The term exemplar is used to refer to the rolled/plain fingerprints in the background database.
} 


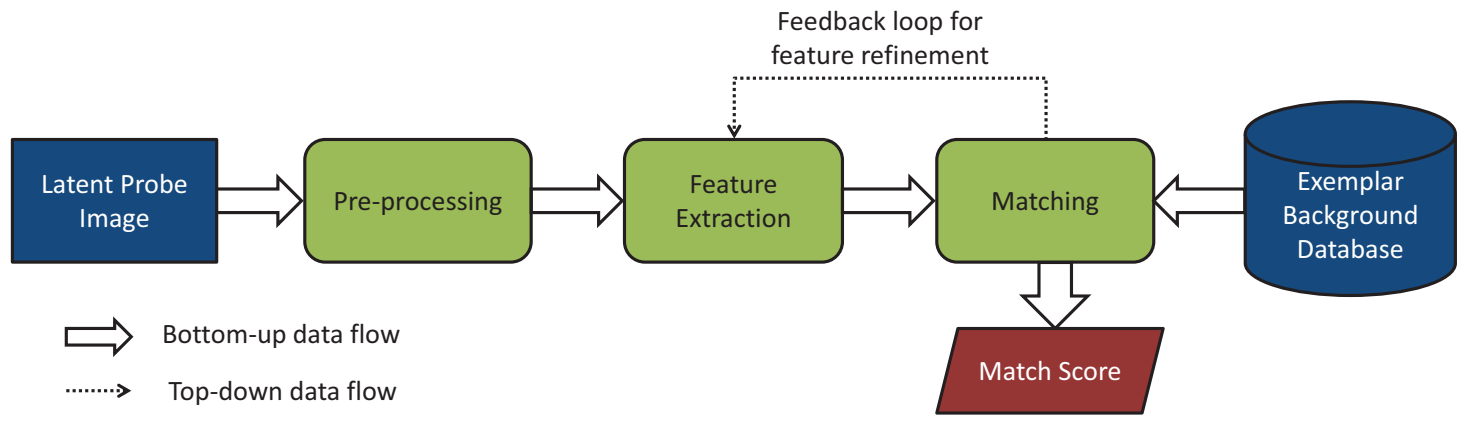

Figure 2. Illustrating the typical bottom-up data flow used in latent to exemplar matching systems. The dotted line shows the feedback path (top-down data flow) in the proposed matching paradigm.

gies (ELFT) [1] conducted by NIST, the Phase-I results showed that the best rank-1 identification accuracy is only $80 \%$ in identifying 100 latent images from amongst a set of 10,000 rolled prints [19]. So, while the Automated Fingerprint Identification Systems (AFISs) work extremely well in matching exemplar fingerprints to each other, there is a considerable performance drop when matching latent fingerprint images to exemplar images. It is generally agreed that latent fingerprint matching is a challenging problem whose performance needs to be significantly improved to reduce the backlog of operational cases in law enforcement agencies. One of the major objectives of FBI's Next Generation Identification (NGI) program [9] is to achieve "lights-out" (fully automatic) capability for latent identification.

In manual matching of latent prints, latent fingerprint examiners usually follow the Analysis, Comparison, Evaluation and Verification (ACE-V) methodology [2]. The ACE$\mathrm{V}$ procedure is a tedious and time consuming process for the latent examiner as it may involve thousands of fingerprint comparisons between different exemplar fingerprint pairs. AFIS is therefore used in the comparison step. Typically, a list of top $K$ matching candidates (with $K$ generally being 20 to 50) is retrieved from the exemplar fingerprint database using a latent matcher, which are then visually inspected by the latent examiner to ascertain the best match. This results in the one of the following three outcomes:

1. The latent examiner correctly matches the latent fingerprint to its true mated exemplar from the candidate list.

2. The examiner erroneously matches the latent fingerprint to another exemplar fingerprint from the candidate list (which is not the true mate).

3. The examiner deems the matching result to be inconclusive because he is unable to find any candidate exemplar that is sufficiently similar to the latent print.

Note that while the second outcome is an erroneous match, the third outcome could be either an error or a reject in the sense that the true mate does not exist in the background database. The proposed feedback matching paradigm is designed to minimize the occurrence of the last two outcomes above by initially retrieving a much larger candidate list (say, $N=200$ ) using the AFIS. Each of these candidates is then viewed as the output of a coarse level match which can be used to refine the features extracted from the latent images. The similarities of these $N$ candidates to the query latent are then recomputed based on the refined latent features to re-rank the candidate list. The latent examiner can then examine the top $K$ candidates $(K \ll N)$ from this re-ranked list for making a better decision during the evaluation step.

State-of-the-art latent matching systems [14], [10], [24], [22] are based on the classical bottom-up matching strategy [8]. The bottom-up approach basically builds a system from several sub-systems or components. In essence, there is a sequential "bottom-up" data flow between the different components of these systems (from preprocessing and feature extraction to matching and match score computation). However, the basic assumption in the bottom-up systems is that if all the individual sub-systems are functioning well, the system as a whole would function well too [7]. In our opinion, this assumption does not hold good for latent matching systems because the feature extraction sub-system does not work sufficiently well for extracting features from operational latents due to the presence of different kinds of structural noise in the latent image [6].

On the other hand, the importance of a feedback mechanism between components or the "top-down" data flow is well known [8]. Bottom-up and top-down approaches have been widely used to model human perception system in cognitive science [12]. Oliver et al. [21] used these strategies to develop a computer vision system for recognizing and modeling human interactions. Top-down approaches have been recently used for object detection and segmentation as well [20], [4]. Feedback mechanisms have also been used for improving the decision making capabilities of artificial neural networks [3]. 
In this paper, we extend this idea of feedback to latent fingerprint matching by incorporating additional top-down data flow between the matching module and feature extraction module (see the dotted line in Figure 2). In the proposed feedback paradigm, feedback from the matching stage is used to refine the features extracted from the latent images. This feedback is particularly useful because features extracted from the latent are often unreliable due to their poor quality. In our opinion, matching latent images based on the initially extracted features without any prior information (bottom-up mode) is prone to error. Additional top-down information flow provided by the feedback allows the matching system to use the hypothesized exemplar mate to guide low level feature extraction in latent and then update the match score accordingly.

In our experiments, we apply the proposed paradigm for automatically refining both the orientation field and the ridge frequency information estimated from the latent images. Experiments conducted on two different latent databases, NIST SD27 [17] and WVU [18], show a marked improvement in the matching accuracies when using the proposed feedback based matching strategy.

\section{Feedback Paradigm for Latent Matching}

The main idea of the proposed paradigm is to generate appropriate feedback from the exemplar fingerprint image for refining the features in the latent image. In essence, feedback from the true mated exemplar should result in a positive refinement of latent features, whereas if it is taken from any impostor mate, it should ideally degrade the initial feature estimates (Figure 3). The initial match between the latent-exemplar pair by a latent matcher is basically what guides this feedback. In other words, the hypothesized match determines the regions where the feedback should be applied as well as the extent or the magnitude of the feedback. The initial matching is also used to filter the background database and generate the initial candidate list. Finally, the candidate list output by the latent matcher is resorted according to the updated match scores based on this feedback.

Let $I^{L}$ be the latent probe image and $I^{R}$ be an exemplar image from the background database. Let $\Theta$ be the set of features extracted from the fingerprint image. Here we denote the feature set corresponding to the latent by $\Theta^{L}$ and that of the exemplar image by $\Theta^{R}$. Typically, feature set $\Theta^{R}$ is pre-computed for each exemplar image. The bottom-up matching process involves matching the two representations $\Theta^{L}$ and $\Theta^{R}$ and assigning the initial match score $\operatorname{Sim}_{I}$ :

$$
\operatorname{Sim}_{I}=S_{I}\left(\Theta^{L}, \Theta^{R}\right) .
$$

Here $S_{I}$ is the similarity function used to generate the match score between the fingerprint feature sets $\Theta^{L}$ and $\Theta^{R}$.

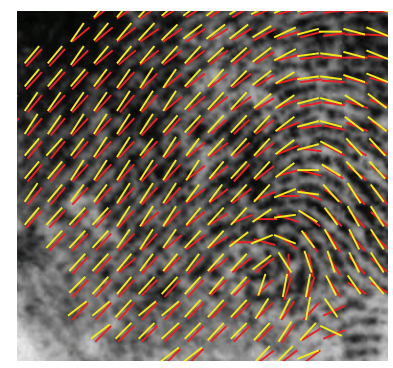

(a)

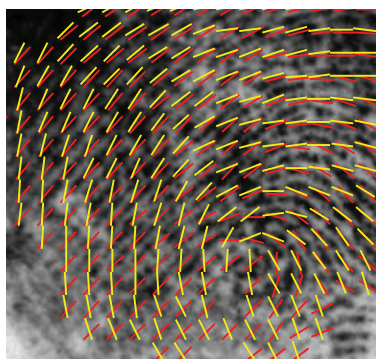

(b)
Figure 3. An example showing the improvement in latent orientation field due to feedback; (a) feedback from true mated exemplar orientation field (in yellow) leads to improved latent orientation field (in red), while (b) feedback from impostor exemplar orientation field (in yellow) degrades latent orientation field (in red). The latent orientation field shown (in red) is manually marked for illustration purposes.

The top $K$ candidate exemplars based on these similarities are retrieved from the background database. Feedback is then provided from the feature set $\Theta^{R}$ of the candidate exemplar image to refine the feature set $\Theta^{L}$ initially computed from the latent image. The refined feature set denoted by $\hat{\Theta}^{L}$ is computed using a function $f$ of the initial feature set $\Theta^{L}$ and the feedback $F$ as follows:

$$
\hat{\Theta}^{L}=f\left(\Theta^{L}, F\right) .
$$

The feedback feature similarity $\operatorname{Sim}_{F}$ between $\hat{\Theta}^{L}$ and $\Theta^{R}$ is then computed using the similarity function $S_{F}$ as follows:

$$
\operatorname{Sim}_{F}=S_{F}\left(\hat{\Theta}^{L}, \Theta^{R}\right) .
$$

Finally, the updated match score $\operatorname{Sim}_{U}$ is calculated from $\operatorname{Sim}_{I}$ and $\operatorname{Sim}_{F}$ using a match score fusion operator $\otimes$ :

$$
\operatorname{Sim}_{U}=\operatorname{Sim}_{I} \otimes \operatorname{Sim}_{F} .
$$

\section{Resorting Candidate List based on Feedback}

The feedback based paradigm is applied for resorting the candidate list generated by the latent matcher proposed in [22]. This matcher is chosen because it is one of the best performing available latent matchers ${ }^{2}$ using minimal human input (uses only marked minutiae for latents). It is referred to as baseline matcher because it is used for initial matching.

The feedback implementation broadly consists of the following four steps (see Figure 4):

\footnotetext{
${ }^{2}$ While we would have liked to use a commercial latent matcher, we have not been able to get access to it. Commercial SDKs such as Verifinger by Neurotechnology (http://www.neurotechnology.com/verifinger.html), do not perform well for latent to exemplar matching since they were not designed for this scenario.
} 


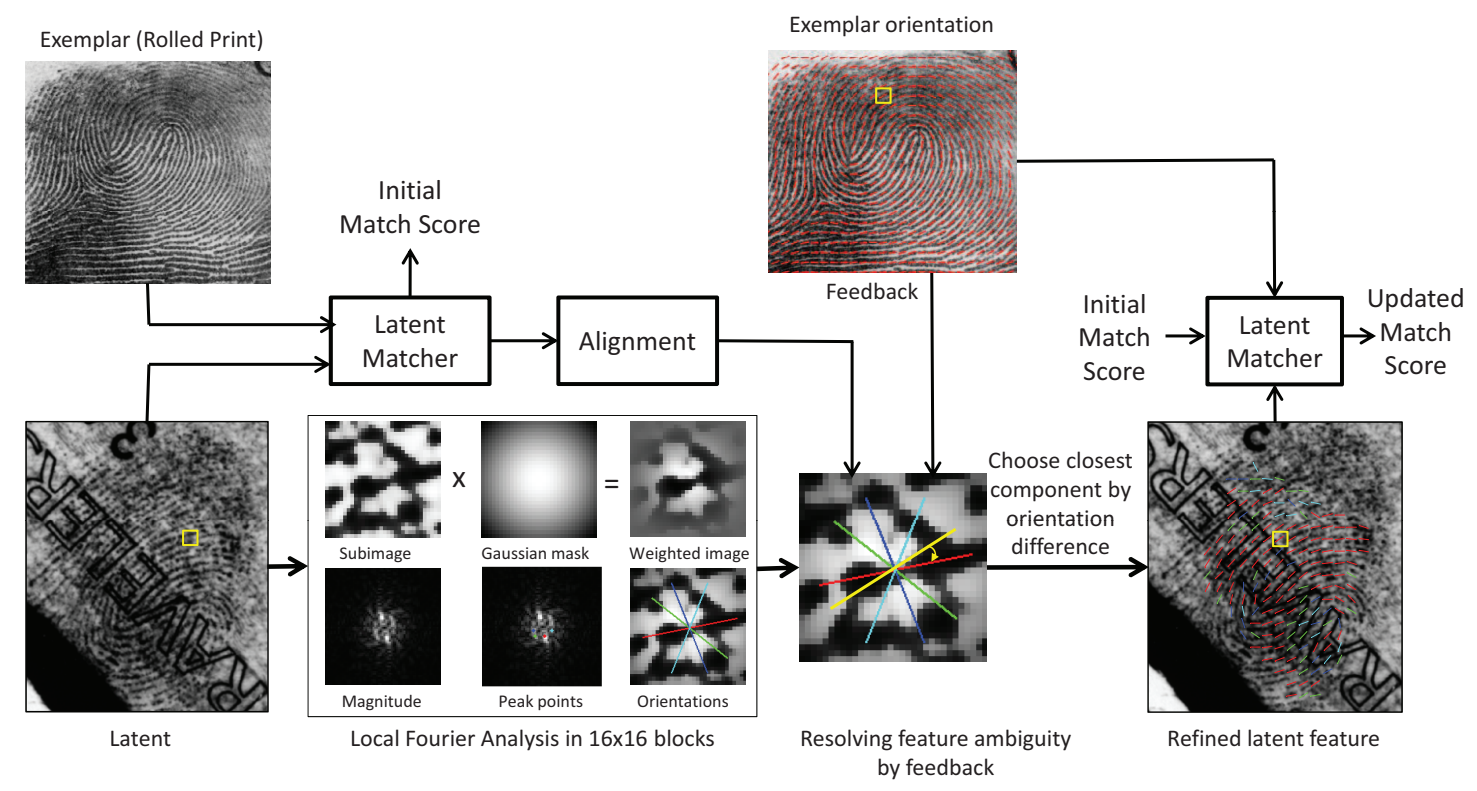

Figure 4. Flow chart illustrating latent fingerprint matching by using feedback from exemplar.

1. Initial Matching and Alignment: The baseline matcher is used to obtain the initial match score, and to generate the minutiae correspondences between the latent and exemplar image. The latent is then aligned to the exemplar image using the scaling, rotation and translation parameters estimated based on these minutiae correspondences.

2. Exemplar Feature Extraction: Ridge orientation and frequency features are extracted from the exemplar.

3. Latent Feature Extraction and Refinement: Latent ridge orientation and ridge frequency features are refined based on the feedback provided from exemplar features.

4. Match Score Computation: Based on the feedback, an updated match score is computed between the latent and the exemplar.

The candidate list is resorted based on the updated match scores between the latent and the retrieved exemplars.

\subsection{Initial Matching and Alignment}

Manually marked minutiae in the latent image and automatically extracted minutiae from the exemplar image (using a commercial off-the-shelf (COTS) matcher) are fed as input to the baseline matcher to obtain the initial match score Sim $_{I}$ and a list of matched minutiae. Let $M^{L}=\left\{\left(x_{i}^{L}, y_{i}^{L}, \theta_{i}^{L}\right) \mid i=1,2, \cdots, P\right\}$ represent the list of matched minutiae for the latent and $M^{R}=$ $\left\{\left(x_{i}^{R}, y_{i}^{R}, \theta_{i}^{R}\right) \mid i=1,2, \cdots, P\right\}$ represent the list of corresponding matched minutiae for the exemplar, where $(x, y)$ are the coordinate values, $\theta$ is the direction of minutia and $P$ is the number of matched minutiae.

Depending on the number of matched minutiae $P$, the transformation $T$ for aligning the latent to the exemplar is estimated differently:

Case I $(P \geq 2)$ : The transformation $T\left(x, y ; a, b, t_{x}, t_{y}\right)$ is estimated by solving the following set of equations:

$$
\left[\begin{array}{l}
x_{i}^{R} \\
y_{i}^{R}
\end{array}\right]=\left[\begin{array}{cc}
a & -b \\
b & a
\end{array}\right]\left[\begin{array}{l}
x_{i}^{L} \\
y_{i}^{L}
\end{array}\right]+\left[\begin{array}{c}
t_{x} \\
t_{y}
\end{array}\right], i=1,2, \cdots, P .
$$

Here, $a=s \cos \Delta \theta$ and $b=s \sin \Delta \theta$, where $s$ is the scale parameter and $\Delta \theta$ is the rotation angle, and $t_{x}$ and $t_{y}$ are the translation parameters. This system of linear equations can be solved by minimizing the least square error.

Now, given the coordinates $\left(x^{L}, y^{L}\right)$ of any point in the latent image, its transformed coordinates $\left(x^{R}, y^{R}\right)$ in the exemplar coordinate system can be obtained by using the transformation $T$ :

$$
\left[\begin{array}{l}
x^{R} \\
y^{R}
\end{array}\right]=\left[\begin{array}{cc}
a & -b \\
b & a
\end{array}\right]\left[\begin{array}{l}
x^{L} \\
y^{L}
\end{array}\right]+\left[\begin{array}{l}
t_{x} \\
t_{y}
\end{array}\right] .
$$

Case II $(P=1)$ : If only one pair of matched minutiae is available, the transformation function is estimated by utilizing both the minutiae location and direction. Let $\left(x_{1}^{L}, y_{1}^{L}, \theta_{1}^{L}\right)$ and $\left(x_{1}^{R}, y_{1}^{R}, \theta_{1}^{R}\right)$ be the matching pair of minutiae in the latent and exemplar, respectively. The rotation angle from latent to exemplar is then estimated by:

$$
\Delta \theta=\theta_{1}^{R}-\theta_{1}^{L} .
$$

Given a point $\left(x^{L}, y^{L}\right)$ in the latent, its transformed coordinates $\left(x^{R}, y^{R}\right)$ in the exemplar coordinate system can be 
calculated by using the transformation $T$ :

$$
\left[\begin{array}{l}
x^{R} \\
y^{R}
\end{array}\right]=\left[\begin{array}{cc}
\cos \Delta \theta & -\sin \Delta \theta \\
\sin \Delta \theta & \cos \Delta \theta
\end{array}\right]\left[\begin{array}{c}
x^{L}-x_{1}^{L} \\
y^{L}-y_{1}^{L}
\end{array}\right]+\left[\begin{array}{l}
x_{1}^{R} \\
y_{1}^{R}
\end{array}\right] .
$$

Note that this transformation does not include any scaling factor because it cannot be estimated based on just a single pair of matched minutiae.

To summarize, translation, scaling and rotation parameters for aligning the latent and the exemplar are estimated based on the matched minutiae pairs. The transformation is then used for transforming the coordinates of each point in the latent to the coordinate system of the exemplar image to align the two images.

\subsection{Exemplar Feature Extraction}

Two different types of local features are computed for the exemplar image, namely ridge orientation and ridge frequency. Given an exemplar image $I^{R}$, its ridge skeleton image $I_{s k}^{R}$ is first extracted using a COTS matcher. The skeleton image is then divided into blocks of size 16 by 16 pixels. Ridge orientation and ridge frequency are then computed for each block $I_{B}^{R}$ in the skeleton image $I_{s k}^{R}$.

\subsection{Latent Feature Extraction and Refinement}

The level one features (e.g. ridge orientation and ridge frequency) in the latent image are very difficult to extract because of the presence of structured noise in the background. Local Fourier analysis is used for this purpose because it has been shown to be resilient to complex background noise [13], [24].

Similar to the exemplar image, the latent image $I^{L}$ is first divided into blocks of size 16 by 16 pixels. For each block $I_{B}^{L}$ in the region of interest (ROI) of the latent, the local ridge orientation and ridge frequency features are then obtained as follows:

1. A $32 \times 32$ sub-image $I_{B^{\prime}}^{L}$ centered at the block $I_{B}^{L}$ is extracted and convolved with a Gaussian filter of the same size with $\sigma=16$.

2. The sub-image $I_{B^{\prime}}^{L}$ is padded with zeros on the borders to get a $64 \times 64$ image $I_{B^{\prime \prime}}^{L}$. This is done to increase the number of sampling points of frequency in the discrete Fourier domain.

3. Fast Fourier Transform (FFT) is applied to the padded sub-image $I_{B^{\prime \prime}}^{L}$. For each peak $(u, v)$ in the magnitude spectrum image, the corresponding orientation $\alpha$ and frequency $f$ is computed by:

$$
\begin{gathered}
\alpha^{L}=\arctan \left(\frac{u}{v}\right), \\
f^{L}=\sqrt{\left(u^{2}+v^{2}\right)} / 64 .
\end{gathered}
$$

4. A set of $L$ peak points $H=\left\{\left(u_{i}, v_{i}\right) \mid i=1,2, \cdots, L\right\}$ of highest magnitude values, and with frequency value satisfying $\frac{1}{16}<f<\frac{13}{64}$, is selected. Note that $L$ is set to 4 in our implementation.

5. The $(x, y)$ coordinates of the central pixel in the block $I_{B}^{L}$ are then transformed to the exemplar coordinate system using the transformation function $T$ estimated previously. Let the transformed coordinates of that pixel be represented as $\left(x^{\prime}, y^{\prime}\right)$.

6. Let $\alpha^{R}$ be the corresponding ridge orientation of the block containing the pixel $\left(x^{\prime}, y^{\prime}\right)$ in the exemplar image. The peak point $l$ (from the set $H$ ) corresponding to the closest ridge orientation to $\alpha^{R}$ from amongst the ridge orientations $\alpha_{i}^{L}$, is then selected as follows:

$$
l=\arg \min _{i} \varphi\left(\alpha_{i}^{L}+\Delta \theta, \alpha^{R}\right),
$$

where $1 \leq i \leq L$ and

$$
\varphi(\alpha, \beta)=\left\{\begin{array}{l}
|\alpha-\beta|, \text { if }|\alpha-\beta|<90 \\
180-|\alpha-\beta|, \text { otherwise }
\end{array}\right.
$$

Here, $\varphi(\alpha, \beta)$ is the function to determine the difference between ridge orientations $\alpha$ and $\beta$ and $\Delta \theta$ is the rotation angle used to align the two ridge orientations $\alpha$ and $\beta$.

7. The ridge orientation and ridge frequency values corresponding to the selected peak point $\left(u_{l}, v_{l}\right)$ are then chosen as the refined ridge orientation and ridge frequency features for the block $I_{B}^{L}$ in latent image.

Note that the refined ridge orientation and ridge frequency features are selected based on the exemplar features, and this essentially is the top-down information flow or the feedback from the exemplar.

\subsection{Match Score Computation}

The overlapping region between the latent and exemplar is first determined using the transformation function $T$. Within the overlapping region, the ridge orientation and ridge frequency similarities $\operatorname{Sim}_{\alpha}$ and $\operatorname{Sim}_{f}$ are then computed as:

$$
\begin{gathered}
\operatorname{Sim}_{\alpha}=\frac{1}{N u m} \sum_{i=1}^{N u m} \exp \left(-\frac{\varphi\left(\alpha_{i}^{L}+\Delta \theta, \alpha_{i}^{R}\right)}{\mu_{\alpha}}\right), \\
\operatorname{Sim}_{f}=\frac{1}{N u m} \sum_{i=1}^{N u m} \exp \left(-\frac{\left|\frac{1}{f_{i}^{L}}-\frac{1}{f_{i}^{R}}\right|}{\mu_{f}}\right)
\end{gathered}
$$

where Num is the number of overlapping blocks; $\alpha_{i}^{L}$ and $\alpha_{i}^{R}$ are the ridge orientations and $f_{i}^{L}$ and $f_{i}^{R}$ are the ridge frequencies of the $i^{\text {th }}$ overlapping block from the latent and exemplar, respectively; $\mu_{\alpha}$ and $\mu_{f}$ are two normalization 


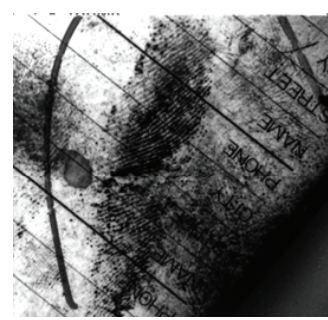

(a)

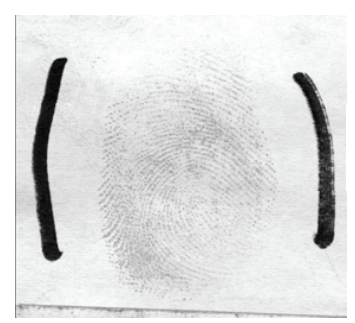

(c)

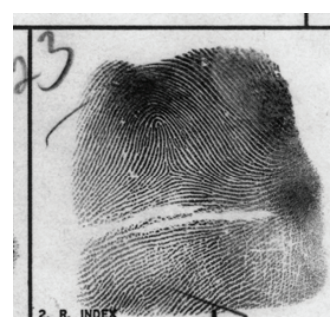

(b)

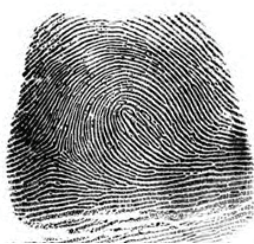

(d)
Figure 5. Sample latent images from (a) NIST SD27 and (c) WVU. Their mated exemplars are shown in (b) and (d), respectively.

parameters which are set to 25 and 5 in our implementation, respectively. The orientation and frequency similarities $\operatorname{Sim}_{\alpha}$ and $\operatorname{Sim}_{f}$ are then combined with the initial match score $\operatorname{Sim}_{I}$ based on product fusion to obtain the updated match score $\operatorname{Sim}_{U}$ as follows:

$$
\operatorname{Sim}_{U}=\operatorname{Sim}_{I} \times \operatorname{Sim}_{\alpha} \times \operatorname{Sim}_{f} .
$$

\section{Experimental Evaluation}

\subsection{Databases}

The proposed feedback paradigm was evaluated on two different latent fingerprint databases: NIST SD27 [17] and WVU [18]. NIST SD27 contains 258 latent images as well as their corresponding exemplar images from operational cases. WVU database, on the other hand, was collected in a laboratory environment. It includes 449 latent images and 4,740 exemplar images out of which 449 exemplars are the true mates of the latents. The original resolution of each fingerprint image in the WVU database is 1000ppi but it was downsampled to 500ppi for our experiments. The two databases have different image characteristics because they have been captured under different environments. The latent images in NIST SD27 contain complex background noise. In contrast, the latent images in the WVU database contain relatively clean background, but the image contrast in WVU database is worse than in NIST SD27 (see Figure $5)$.

To simulate the real world scenario of latent matching, 27,000 exemplar images from NIST SD14 [16] database were augmented with the exemplars in NIST SD27 and WVU to form a background database of 31,998 exemplar

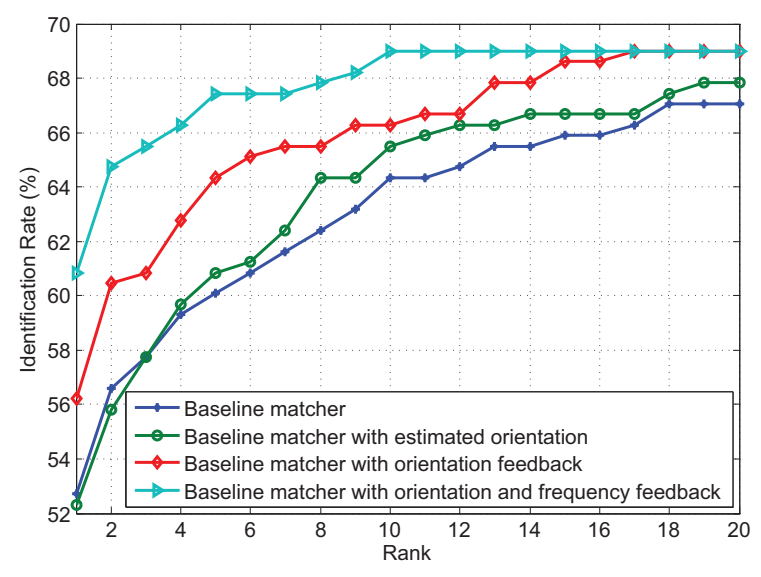

Figure 6. Performance of baseline matcher [22], baseline matcher fused with estimated orientation field [10], and baseline matcher with proposed orientation field and frequency map feedback on the NIST SD27 database.

images. The baseline matcher [22] matches each latent against the background database and returns a candidate list of 200 exemplars. The proposed feedback paradigm is then applied on the candidate list to update the match scores and resort the candidate list as described in Section 3.

\subsection{Performance on NIST SD27 Database}

The Cumulative Match Characteristics (CMC) curves shown in Figure 6 illustrate the performance of baseline matcher with and without feedback on NIST SD27. The baseline matcher in [22] has a rank-1 identification accuracy of 53\%. However, since it only uses minutiae information and no other information in the latent, we fuse the baseline matcher with a state-of-the-art orientation field estimation algorithm for latents [10]. This additional orientation field information improves the performance of the baseline matcher only marginally. However, using the proposed orientation field feedback to refine the latent features improves the rank-1 identification accuracy by around $3 \%$. Incorporating ridge frequency along with orientation field in the feedback results in an additional improvement of 5\% in the rank-1 identification accuracy. Thus, an overall gain of around $8 \%$ in the rank-1 identification accuracy over the baseline matcher is achieved on the NIST SD27 database using the proposed feedback paradigm.

Figure 7 shows the effect of feedback on various quality latents in NIST SD27 (good, bad and ugly). The baseline matcher, as expected, performs the best for good quality latent images, and the worst for the ugly quality latents. Interestingly, the proposed feedback paradigm provides the highest performance gain of around $12 \%$ for the ugly quality latents. This is because the reliability of features initially extracted from ugly quality latents is significantly lower as 


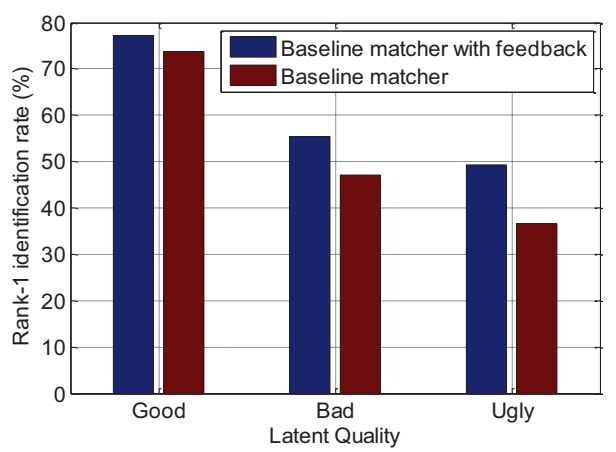

Figure 7. Rank-1 identification rates of baseline matcher [22] with and without orientation field and frequency map feedback for "Good", "Bad" and "Ugly" quality latents in NIST SD27 database.

compared to good quality latents, and hence it is the poor quality latents that benefit the most from the feedback. For bad quality latents, the rank-1 identification accuracy improves by around $8 \%$ whereas for the good quality latents, the improvement is only about $3 \%$.

Figure 8 shows two examples where the retrieval rank of mated prints is significantly improved by applying feedback orientation, whereas Figure 9 shows an example where the retrieval rank of mated print degrades after matching with feedback orientation. The matching performance generally degrades when (i) the ridge structure of the impostor is similar to latent and (ii) the impostor exemplar is of better quality as compared to the true mate resulting in better quality features being extracted from the impostor.

\subsection{Performance on WVU Database}

The Cumulative Match Characteristics curves (Figure 10) for the WVU database also demonstrate the advantage of using the proposed feedback framework with the baseline matcher. A performance gain of around 3\% in the rank-1 identification accuracy is observed with the frequency map and orientation field feedback. Note that the improvement is smaller as compared to NIST SD27 because the contrast of latents in WVU is in general poor, and hence the level one features are difficult to extract in the frequency domain.

\subsection{Computational Complexity}

The current implementation of the feedback paradigm uses local ridge orientation and ridge frequency features extracted at multiple peak points in the frequency representation of the latent image as described in Section 3. To reduce the computational complexity, these features are computed only once for each query, and then used in matching against all exemplar candidates. Further, the feedback mechanism does not involve the entire exemplar database but is used only to re-rank the top 200 candidates returned by the baseline matcher.

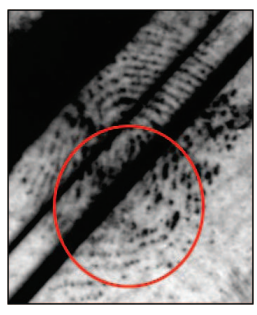

(a)

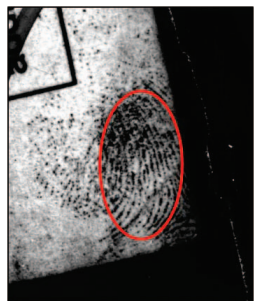

(d)

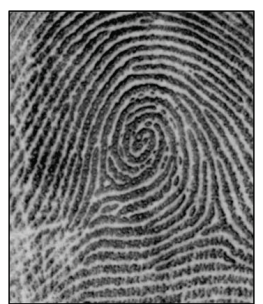

(b)

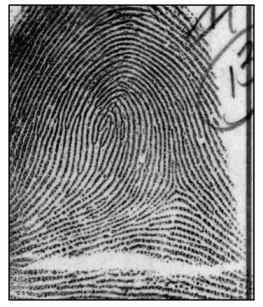

(e)

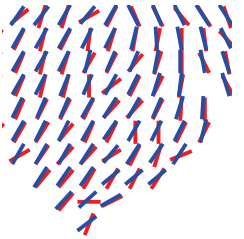

(c)

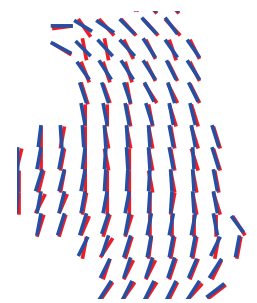

(f)
Figure 8. Successful examples of matching by feedback; (a) and (d) are latents in NIST SD27 (B117 and B182); (b) and (e) are mated prints of (a) and (d), respectively, and (c) and (f) are the overlay of latent orientation fields (in red) of (a) and (d) estimated after feedback from mated exemplar image with exemplar orientation fields (in blue). The orientation fields are shown within the region indicated by the red ellipses in latents. The retrieval rank of the mated print of latent (a) was improved from 42 to 2 and that of (d) was improved from 42 to 3 after matching with orientation field feedback.

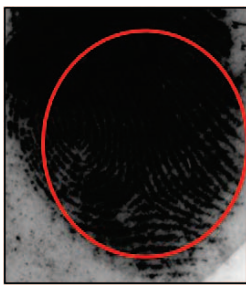

(a)

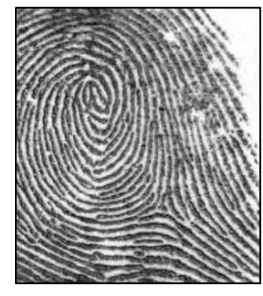

(b)

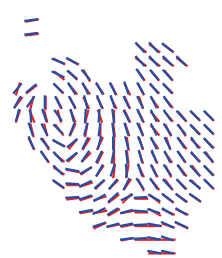

(c)
Figure 9. An unsuccessful example of matching by feedback; (a) a latent in NIST SD27 (G044), (b) impostor exemplar image, and (c) overlay of latent orientation (in red) after feedback from impostor exemplar image and exemplar orientation field (in blue). The retrieval rank of this impostor exemplar improved from 29 to 5 after matching with feedback orientation field, while, the rank of true mated print degraded from 6 to 27 after matching with orientation field feedback.

The algorithm has been implemented in MATLAB and runs on a desktop system with Intel $\AA$ Core $^{\mathrm{TM}} 2$ Duo CPU of $2.93 \mathrm{GHz}$ and $4.00 \mathrm{~GB}$ of RAM with Windows $7 \mathrm{Op}-$ erating system. For the NIST SD27 database, the average time to extract local orientation and frequency features for a latent is about $0.74 \mathrm{sec}$ and the average time to match a latent against the 200 candidates is about $4 \mathrm{sec}$. The extra 


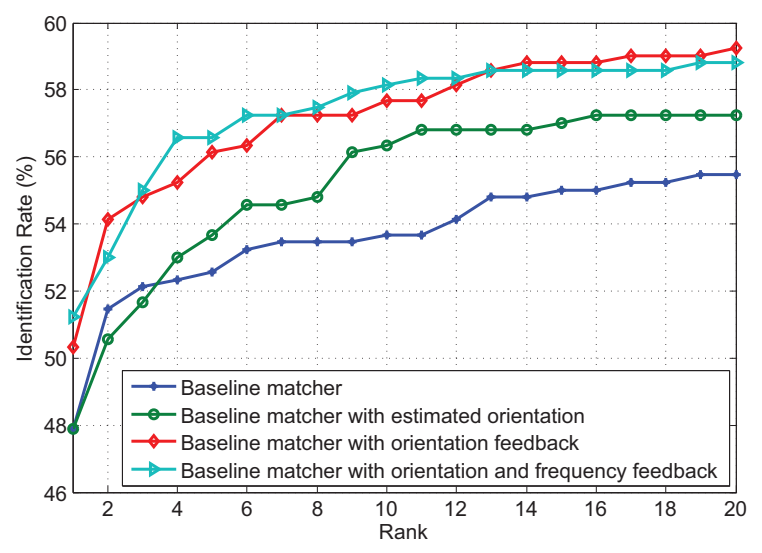

Figure 10. Performance of baseline matcher [22], baseline matcher fused with estimated orientation field [10], and baseline matcher with proposed orientation field and frequency map feedback on the WVU database.

computational cost incurred in matching the latent is worth the significant improvement in performance, especially in forensic applications which demand high latent matching accuracy.

\section{Conclusions}

Given the relatively poor quality of operational latent images, feature extraction is one of the major challenges for a latent matching system. To deal with complex background noise in the latent, we propose incorporating feedback from exemplar to refine feature extraction in latent with the eventual goal of improving the latent matching accuracy. The proposed feedback paradigm has been used to refine the latent orientation field and frequency map and to resort the candidate list returned by a latent matcher. Experimental results show significant improvement in the latent matching accuracy using the feedback mechanism, especially for poor quality latents.

The matching accuracy can be further improved by: (i) including additional features in the feedback system, e.g. ridge skeleton and minutiae; (ii) improving the similarity computation between refined latent features and exemplar features. The proposed feedback paradigm can be wrapped around any latent matcher to improve its matching performance.

\section{References}

[1] Evaluation of latent fingerprint technologies. http:// fingerprint.nist.gov/latent/elft07/, 2007.

[2] D. Ashbaugh. Quantitative-Qualitative Friction Ridge Analysis: An Introduction to Basic and Advanced Ridgeology. CRC Press, 1999.

[3] M. Bodén. A guide to recurrent neural networks and backpropagation. The DALLAS project. Report from the NUTEK- supported project AIS-8, SICS. Holst: Application of data analysis with learning systems, (2):1-10, 2001.

[4] E. Borenstein and S. Ullman. Combined top-down/bottomup segmentation. IEEE TPAMI, 30(12):2109-2125, 2008.

[5] C. Champod, C. Lennard, P. Margot, and M. Stoilovic. Fingerprints and Other Ridge Skin Impressions. CRC Press, 2004.

[6] H. Choi, M. Boaventura, I. Boaventura, and A. Jain. Automatic segmentation of latent fingerprints. In IEEE Fifth International Conference on Biometrics: Theory, Applications and Systems, pages 303 -310, 2012.

[7] B. Coppin. Artificial Intelligence Illuminated. Jones \& Bartlett Learning, 2004.

[8] R. Duda, P. Hart, and D. Stork. Pattern Classification. John Wiley and Sons Inc., second edition, 2001.

[9] FBI. Next Generation Identification. http://www. fbi.gov/about-us/cjis/fingerprints_ biometrics/ngi, 2012.

[10] J. Feng, J. Zhou, and A. Jain. Orientation field estimation for latent fingerprint enhancement. IEEE TPAMI (to appear), 2012.

[11] F. Galton. Finger Prints. MacMillan, 1892.

[12] J. Hollands and C. Wickens. Engineering Psychology and Human Performance. Prentice Hall, New Jersey, 1999.

[13] A. Jain and J. Feng. Latent palmprint matching. IEEE TPAMI, 31(6):1032-1047, 2009.

[14] A. Jain and J. Feng. Latent fingerprint matching. IEEE TPAMI, 33(1):88-100, 2011.

[15] H. Lee and R. Gaensslen. Advances in Fingerprint Technology. CRC Press, 2001.

[16] NIST Special Database 14. http://www.nist.gov/ srd/nistsd14.cfm.

[17] NIST Special Database 27. http://www.nist.gov/ srd/nistsd27.cfm.

[18] West Virginia University (WVU) Marked rolled prints database. http://www. csee.wvu.edu/ ross/i-probe/.

[19] NIST. Summary of results from elft07 phase i testing. http://fingerprint.nist.gov/latent/ elft07/phase1_aggregate.pdf, 2007.

[20] A. Oliva, A. Torralba, M. Castelhano, and J. Henderson. Topdown control of visual attention in object detection. In Proceedings of International Conference on Image Processing, volume 1, pages I-253. IEEE, 2003.

[21] N. Oliver, B. Rosario, and A. Pentland. A bayesian computer vision system for modeling human interactions. IEEE TPAMI, 22(8):831-843, 2000.

[22] A. Paulino, J. Feng, and A. Jain. Latent fingerprint matching using descriptor-based hough transform. IEEE TIFS, 8(1):31-45, 2013.

[23] C. Wilson. Fingerprint vendor technology evaluation 2003: Summary of results and analysis report, nistir 7123. http://fpvte.nist.gov/report/ir_ 7123_analysis.pdf, 2004.

[24] S. Yoon, J. Feng, and A. Jain. On latent fingerprint enhancement. In SPIE Conference Series, volume 7667, pages 766707_01-766707_10, 2010. 